\title{
Actividad física en época de confinamiento por COVID-19 para reducir los niveles de estrés en estudiantes universitarios
}

\section{Physical activity in time confinement due to COVID-19 to reduce stress levels in university students.}

\author{
MAPE. María Julia León Bazán ${ }^{1}$, Dra. María Lizett Zolano Sánchez ${ }^{2}$ \\ 1julia.leon@unison.mx, ORCID: 0000-0002-7405-6141, Universidad de Sonora, Unidad Centro. \\ ²maria.zolano@unison.mx, ORCID: 0000-0001-7526-2038, Universidad de Sonora, Unidad Centro.
}

Dol https://doi.org/10.46589/rdiasf.vi35.394

Recibido 2 de mayo 2021.

Aceptado 2 de junio 2021

Publicado 30 de junio de 2021

\section{Resumen}

Esta investigación se realizó bajo un enfoque metodológico descriptivo y exploratorio, tomando como referencia, estudiantes inscritos en el semestre 2020-2 de la Licenciatura en Cultura Física y Deporte de la Universidad de Sonora, Unidad Centro y de la Licenciatura en Educación de la Unidad Santa Ana, y que están en confinamiento debido a COVID-19. El objetivo de este proyecto de investigación es analizar la posible relación entre los niveles de estrés percibido por estudiantes universitarios que atraviesan época de confinamiento por COVID-19 y, determinar como la actividad física influye en la mejora de la salud física y psicológica durante la pandemia. Se aplicó un cuestionario estandarizado donde se analizaron dos variables: Variable 1; Estrés percibido en el estudiante universitario durante el confinamiento provocado por el COVID-19 y, Variable 2; Actividad Física y Salud. Los principales resultados muestran en la variable 1, que los estudiantes no están preparados para trabajar desde casa, no cuentan con los medios ni la preparación técnica para hacerlo y el tiempo que tienen en confinamiento les causa cansancio, ansiedad y estrés por no tener actividad física. En la variable 2, el confinamiento les ha llevado a aislarse de la familia y los amigos porque invierten muchas horas a las actividades académicas, el sedentarismo y descuidan sus hábitos alimenticios, lo que les ha provocado cansancio que aunado a la falta de actividad física les genera estrés y ansiedad, poniendo en riesgo su salud física y mental. Como conclusión final, la realización de actividad física, puede ayudar a mejorar la salud psicológica del estudiante, de tal forma que pueda ver reflejado en su rendimiento académico.

Palabras Clave: Actividad Física, Estudiantes, COVID-19, Estrés. 


\begin{abstract}
This research was carried out under a descriptive and exploratory methodological approach, taking as a reference, students enrolled in the 2020-2 semester of the Bachelor's Degree in Physical Culture and Sports at the University of Sonora, Central Unit and the Bachelor's Degree in Education from the Santa Unit Ana, and that they are in lockdown due to COVID-19. The objective of this research project is to analyze the possible relationship between the levels of stress perceived by university students who are going through a period of confinement due to COVID-19 and to determine how physical activity influences the improvement of physical and psychological health during the pandemic. A standardized questionnaire was applied where two variables were analyzed: Variable 1; Perceived stress in the student university during confinement caused by COVID-19 and, Variable 2; Physical activity and health. The principal results show in variable 1, that the students are not prepared to work from home, they do not have the means or the technical preparation to do so and the time they have in confinement causes them tiredness, anxiety and stress because they do not have physical activity. In variable 2 , confinement has led them to isolate themselves from family and friends because they invest many hours in academic activities, sedentary lifestyle and neglect their eating habits, which has caused them tiredness that, together with the lack of physical activity generates stress and anxiety, putting your physical and mental health at risk. As a final conclusion, the realization of physical activity, can help to improve the psychological health of the student, in such a way that it could be reflected in their academic performance.
\end{abstract}

Key words: Physical Activity, Students, COVID-19, Stress.

\title{
Introducción
}

El confinamiento ha sido una de las estrategias de salud pública internacionales para detener la propagación del COVID-19, la cual consiste en el aislamiento social y resguardo de las personas en sus hogares, implicando un cambio drástico en las actividades y en los comportamientos en la vida cotidiana, entre ellos, el trabajo y la educación en línea, así como las restricciones en la práctica de actividades fuera de casa, obligando a los ciudadanos a implementar nuevas formas de vida dentro del hogar ante esta contingencia (World Health Organization, 2020).

El confinamiento puede tener efectos negativos en la salud de las personas mayores; pero no solo en ellos, también en los estudiantes universitarios, que pueden ver cómo su salud, su calidad de vida o el rendimiento académico disminuyen, en parte, debido a la baja actividad física, porque en México, de inmediato se prohibió hacer ejercicio al aire libre desde el inicio de pandemia el 14 
de marzo del 2020, cuando se declaró el estado de alarma para la gestión de la situación de crisis sanitaria ocasionada por la COVID-19, la reducción de oportunidades para realizar las tareas que anteriormente eran consideradas como comunes o cotidianas durante el confinamiento puede tener efectos adversos a corto, medio y largo plazo en los estudiantes (Books et al., 2020; Giraldo, 2021)

Existen estudios científicos en diferentes países, que demuestran que el confinamiento durante una pandemia provoca impacto psicológico en las personas, donde se refleja la desesperación, la ansiedad y el enojo (Ko et al., 2006; Yoon et al., 2016). Más recientemente, en España, Ozamiz-Etxebarria et al. (2020), condujeron una investigación con el objetivo de determinar los niveles de estrés, ansiedad y depresión en la primera fase del brote de Covid-19 con una población conformada por 976 ciudadanos del norte de España; dichos investigadores detectaron que los grupos más jóvenes de la población en estudio (18-25 años) presentaron promedios más altos en estrés, ansiedad y depresión en comparación a los grupos de 26-60 años y mayores de $60 \mathrm{y}$, el impacto psicológico fue más alto en las personas con enfermedades crónicas.

En tiempos de confinamiento por causa de pandemia, la prioridad está centrada en la búsqueda de una estabilidad financiera que ayude a las familias a contar con alimentos para sobrevivir, evitando estar en contacto con muchas personas, pero también es importante considerar que en esta parte del proceso también aumenta el tiempo libre, el sedentarismo y, las personas dejan de hacer actividades físicas continuas de alto o bajo rendimiento causando estrés, ansiedad y depresión (Rodríguez et al., 2020; Ozamiz, Dosil, Picaza, \& Idoiaga, 2020).

La pandemia del COVID-19 cambio radicalmente las actividades normales de las personas alrededor del mundo, llegando a tal punto de obligar a un confinamiento social dentro de los hogares para prevenir la salud y la vida de las familias y, el estilo de vida se modificó de manera especial entre los estudiantes, quienes vieron transformadas sus actividades escolares a una interacción virtual en sus clases y en el caso de los estudiantes universitarios la falta de la Actividad Física (AF) presento problemas de salud, tanto físico como psicológico, ya que en esta etapa se acostumbra la práctica deportiva como por ejemplo caminatas, ejercicios al aire libre y en gimnasios (Villaquiran, Ramos, Jácome, y Meza, 2020; Ruiz y Gómez, 2021).

La OMS sostiene que, el comportamiento sedentario y los niveles bajos de Actividad Física pueden producir efectos negativos en la salud, la calidad de vida y el bienestar de las 
personas, en tanto que la Actividad Física y las técnicas de relajación resultan ser herramientas excelentes para ayudar a mantener la calma y proteger su salud durante el aislamiento de cuarentenas (OMS, 2020). Con base en lo anterior, se considera una estrategia preventiva y altamente efectiva para la Universidad de Sonora, el crear programas donde se incluya la actividad física para lograr calidad de vida durante el confinamiento. El objetivo de este proyecto de investigación es analizar la posible relación entre los niveles de estrés percibido por estudiantes universitarios que atraviesan época de confinamiento por COVID-19 y, determinar como la actividad física influye en la mejora de la salud física y psicológica durante la pandemia.

\section{Materiales y Método}

En este documento se presentan los resultados de una investigación de campo bajo un enfoque metodológico de tipo descriptiva y exploratoria. La población de estudio seleccionada para esta investigación fueron un total de 180 estudiantes de la Universidad de Sonora, México. Se utiliza un muestreo no probabilístico y la muestra está constituida por 60 estudiantes de la Licenciatura en Cultura Física y Deporte de la Unidad Centro y 60 de la Licenciatura en Educación de la Unidad Santa Ana. Se tomaron en consideración criterios de inclusión tales como: estar inscrito de forma presencial en las licenciaturas de las unidades elegidas para el estudio. Aceptar participar de manera voluntaria y asentir el consentimiento informado. Para este estudio se aplicó un cuestionario estandarizado (validado a juicio de expertos y con un coeficiente de confiabilidad Alpha de Cronbach de 0,92 Muy Alta) por (González-Díaz y Perez, 2015), donde se analizan dos variables: Variable 1; Estrés percibido por el estudiante universitario durante el confinamiento provocado por el COVID-19; (determina la percepción de estrés psicológico, el cómo las situaciones que se presentan día a día pueden ser apreciadas como estresantes); Variable 2; Actividad Física y Salud. Posteriormente e aplicó a estudiantes con las características planteadas para este estudio mediante un Google form (González-Díaz et al., 2016).

\section{Resultados}

Se presentan a continuación los resultados más relevantes de los avances de la investigación basados en el objetivo central de esta investigación. 


\subsection{Tabulación, gráficas, interpretación y discusión de los resultados}

\subsubsection{Variable 1; Estrés percibido por el estudiante universitario durante el confinamiento} provocado por el COVID-19

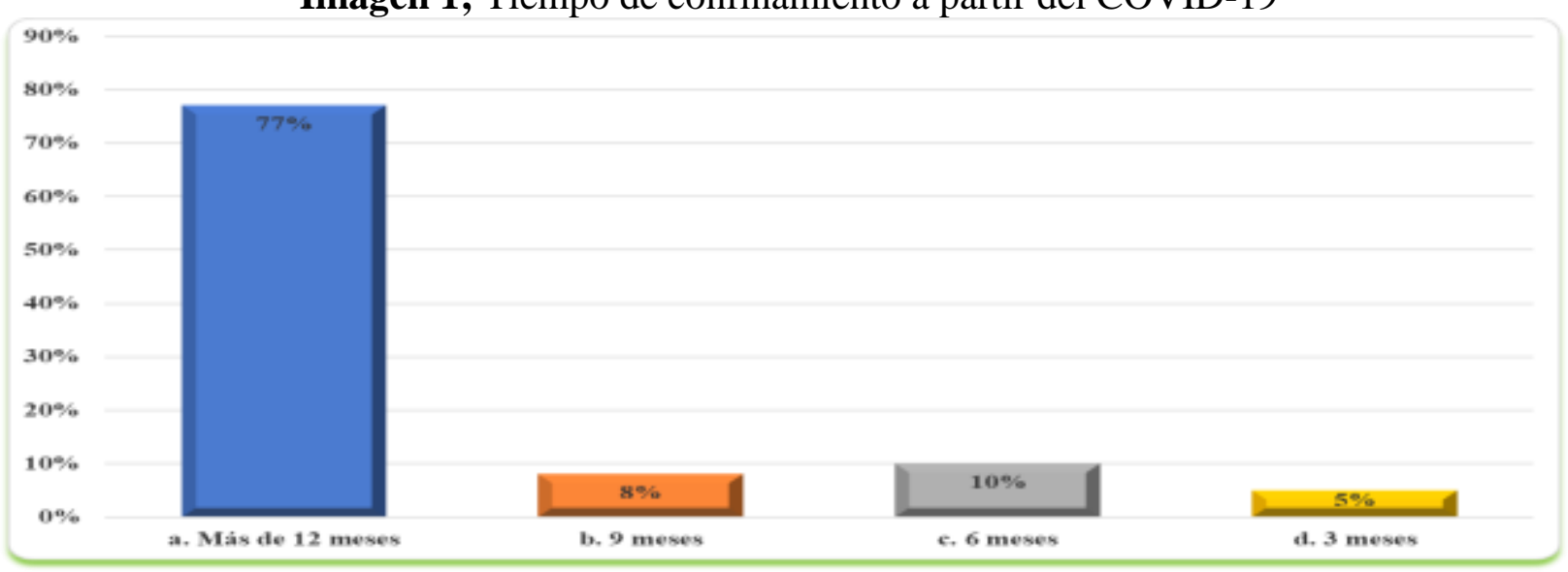

Fuente: Elaboración propia con base a los resultados estadísticos.

En la Imagen 1, se muestra el tiempo de confinamiento a partir del COVID-19 y se observa que el $77 \%$ de los alumnos de la muestra analizada tienen más de 12 meses de confinamiento, un $10 \%$ tiene 6 meses, un $8 \%$ tiene 9 meses y un 5\% cuenta con 3 meses de confinamiento.

\section{Imagen 2}

2; Tiempo diario dedicado a los espacios virtuales por causa de actividades académicas

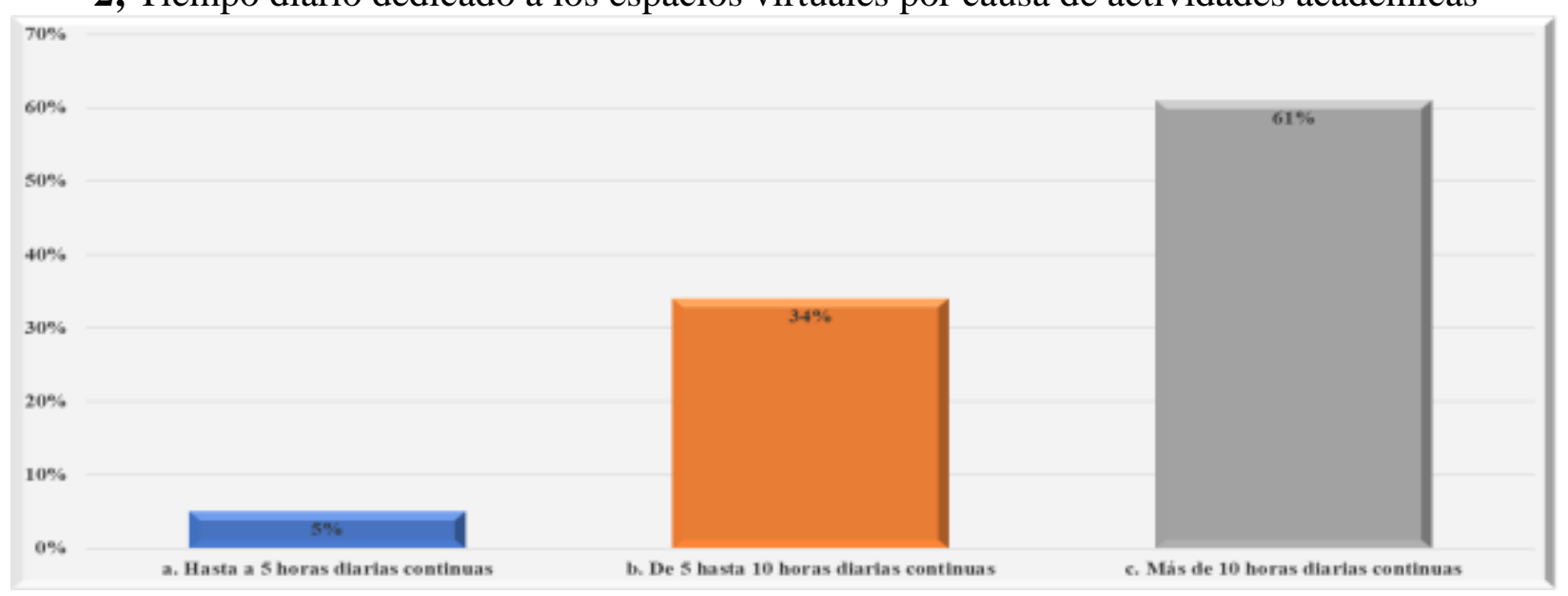

Fuente: Elaboración propia con base a los resultados estadísticos. 
En la Imagen 2, se muestra el tiempo diario dedicado a los espacios virtuales por causa de actividades académicas durante el confinamiento a partir del COVID-19 y se observa que el $61 \%$ de los alumnos de la muestra analizada dedica más de 10 horas diarias continuas, un 34\% dedica de 5 a 10 horas diarias continuas y un $5 \%$ hasta 5 horas diarias continuas.

Imagen 3; Ha trabajado con espacios virtuales antes de la pandemia COVID-19

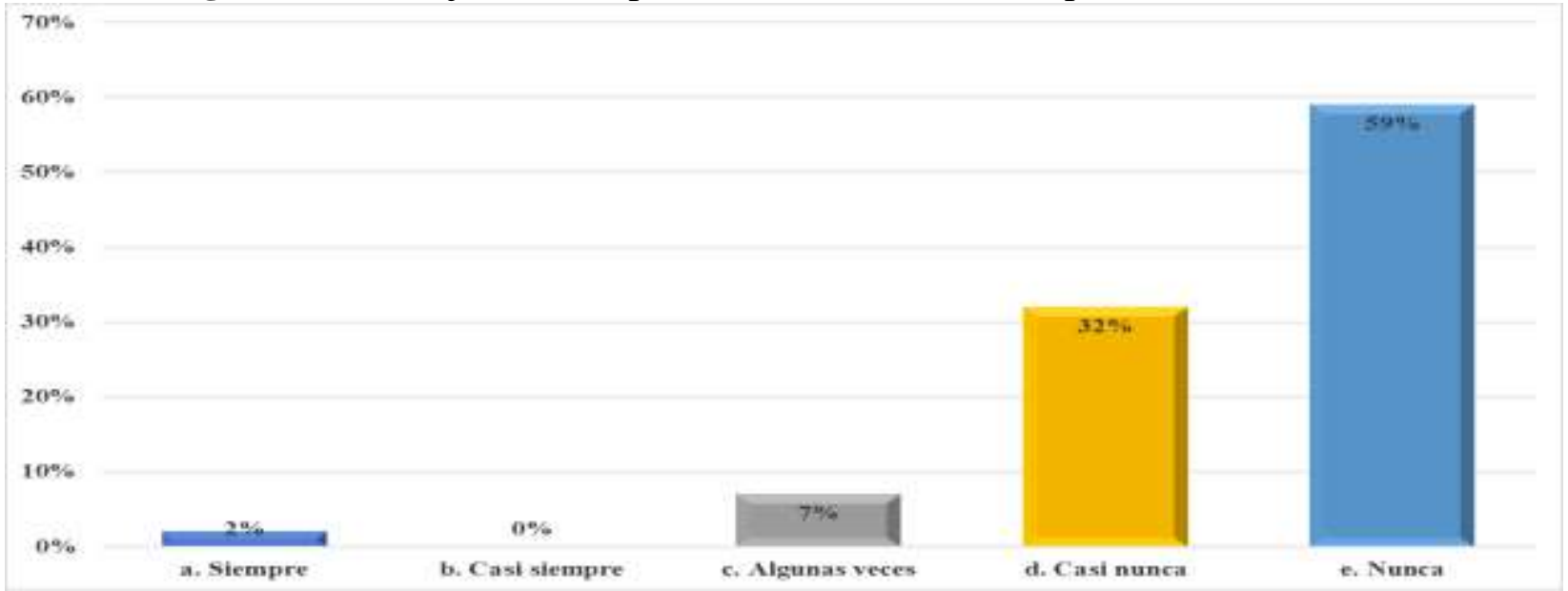

Fuente: Elaboración propia con base a los resultados estadísticos.

En la Imagen 3, se puede observar que el 59\% nunca ha trabajado con espacios virtuales antes de la pandemia COVID-19, el 32\% casi nunca lo ha hecho, un 7\% algunas veces y solo el $2 \%$ menciona que siempre lo ha hecho.

Imagen 4; Aspectos que influyen de forma negativa el desempeño académico durante el confinamiento 


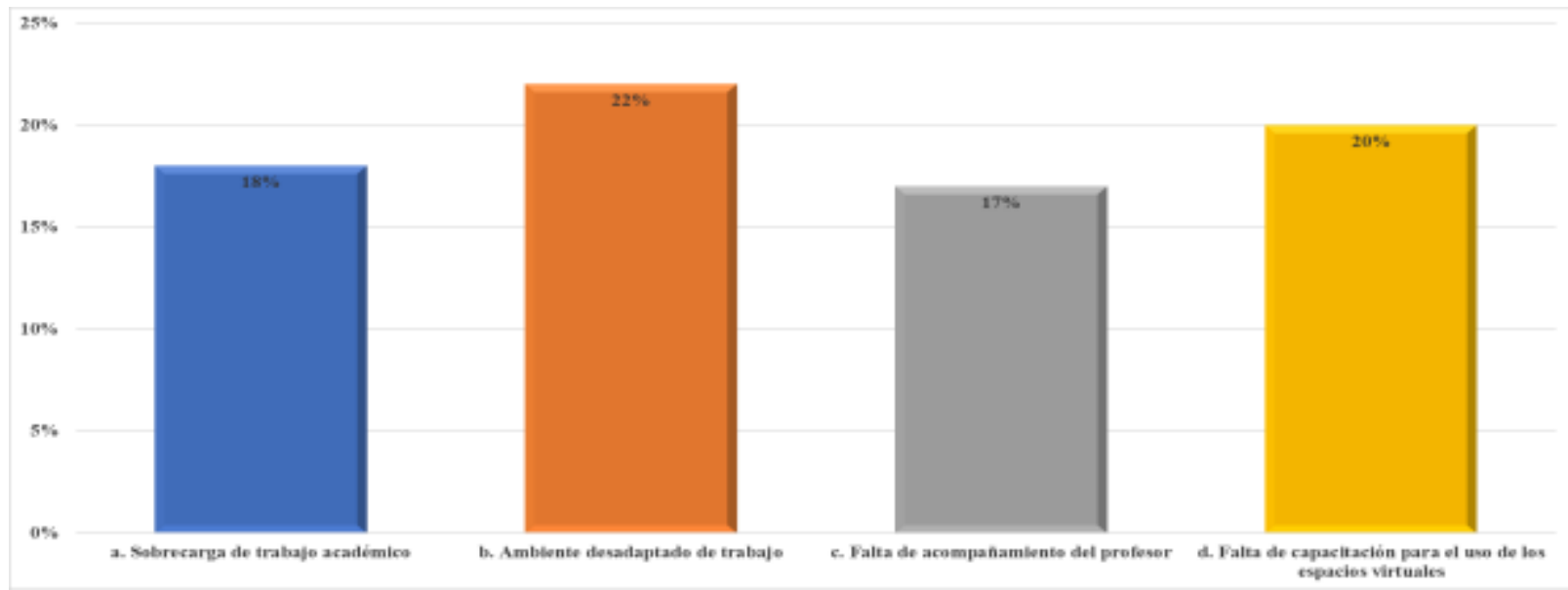

Fuente: Elaboración propia con base a los resultados estadísticos.

En la Imagen 4, se muestra las percepciones de los estudiantes con el fin de indagar sobre aspectos que influyen de forma negativa en su desempeño académico durante el confinamiento a partir del COVID-19. Las respuestas muestran que para el 22\% influye el ambiente desadaptado del área de trabajo, para el 20\% la falta de capacitación en el uso de los espacios virtuales, para el $18 \%$ la sobrecarga de trabajo académico y para el $17 \%$ la falta de acompañamiento del profesor.

.Imagen 5. Espacios de convivencia social y familiar durante la pandemia COVID-19

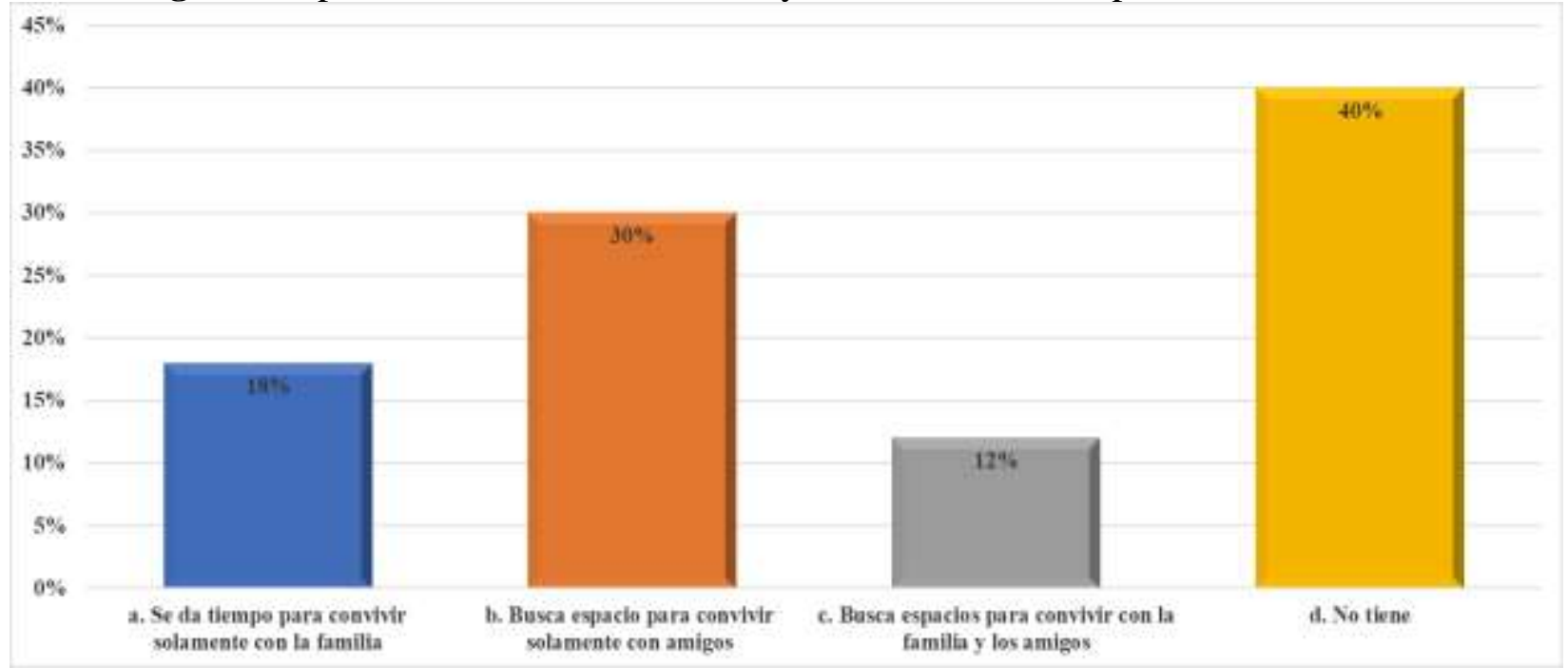

Fuente: Elaboración propia con base en los resultados obtenidos. 
En la Imagen 5, se relaciona con los espacios de convivencia social y familiar durante la pandemia COVID-19. El 40\% no los tiene, el 30\% busca espacios para convivencia solo con los amigos, el $18 \%$ busca espacios de convivencia solamente con la familia y el $12 \%$ se da tiempo para convivir con la familia y los amigos.

Imagen 6. Principal preocupación durante el confinamiento a partir del COVID-19

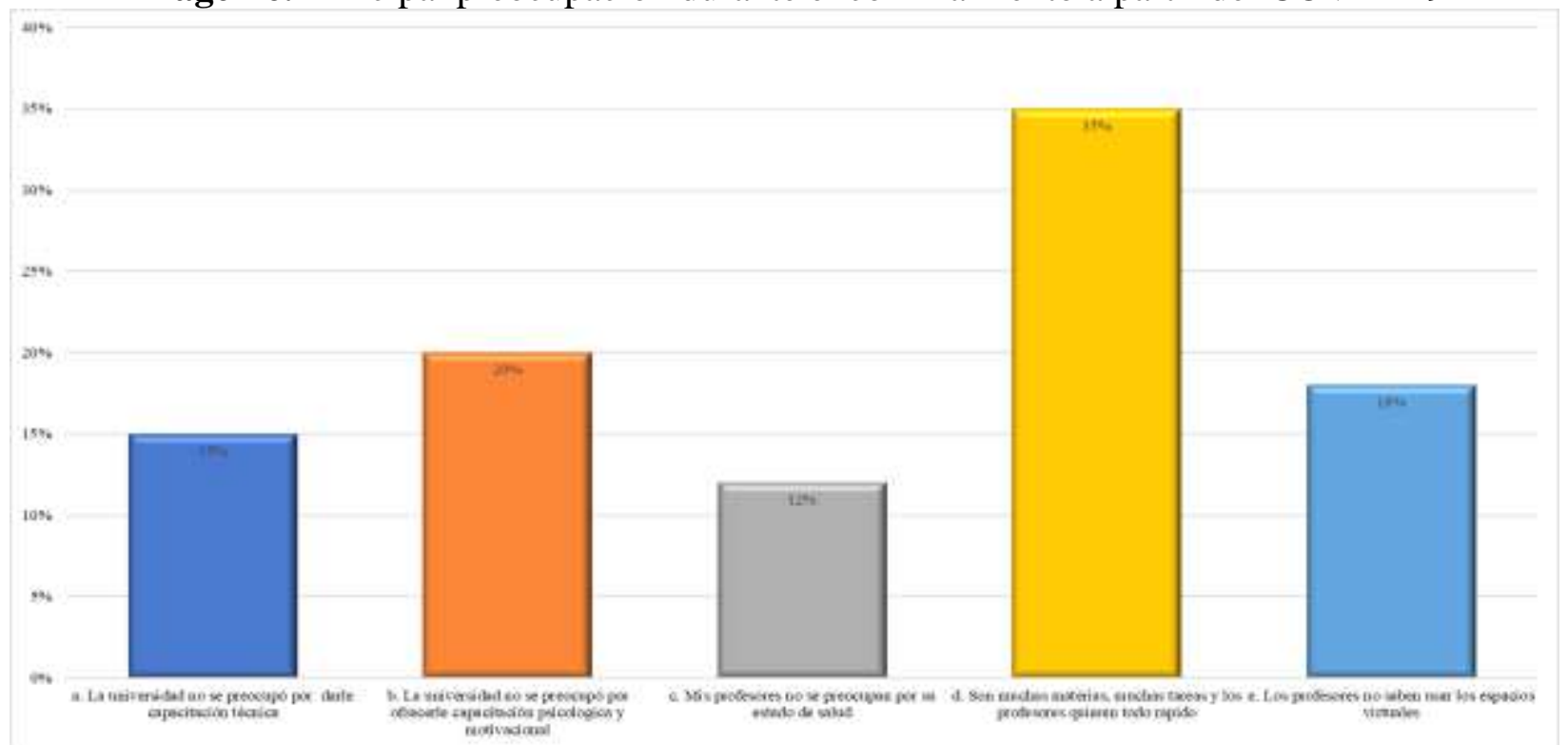

Fuente: Elaboración propia con base en los resultados obtenidos.

En la Imagen 6, se relaciona con la principal preocupación durante el confinamiento a partir del COVID-19. Para el 35\% su principal preocupación es que son muchas tareas y los profesores quieren todo rápido, para el $20 \%$ su principal preocupación es que la universidad no se 
preocupó por ofrecerles capacitación psicológica y motivacional, al 18\% le preocupa que los profesores no saben usar los espacios virtuales, el 15\% se preocupa porque la universidad no se preocupó por darle capacitación técnica y al 12\% les preocupa que sus profesores no se preocupan por su estado de salud.

Imagen 7. Que te genera estrés y ansiedad durante el confinamiento

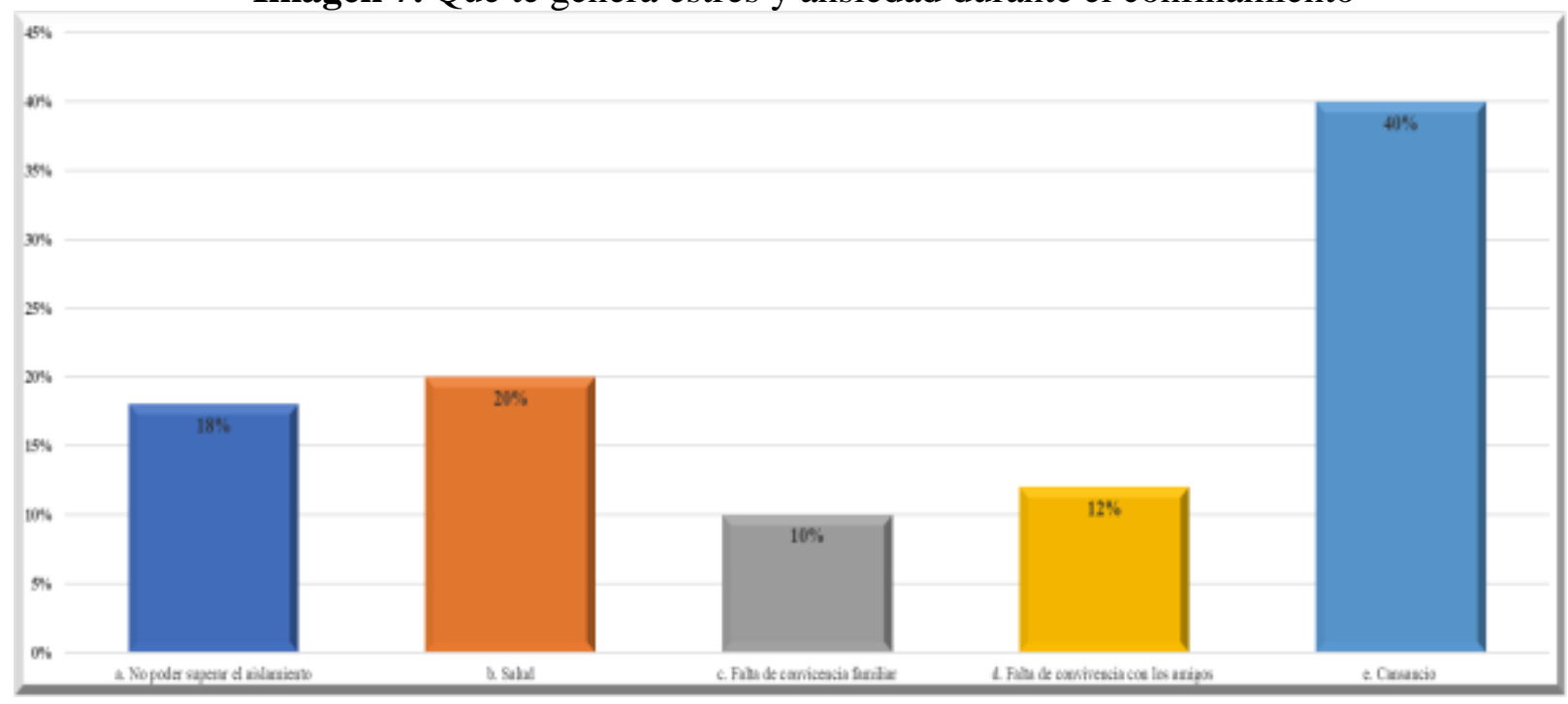

Fuente: Elaboración propia con base en los resultados obtenidos.

En la Imagen 7, se relaciona con las principales causas que le generan estrés y ansiedad al estudiantes durante el confinamiento a partir del COVID-19. Para el $40 \%$ el cansancio le genera estrés y ansiedad, para el $20 \%$ la salud, para el $18 \%$ no poder superar el aislamiento, para el $12 \%$ la falta de convivencia con los amigos y para el $10 \%$ la falta de convivencia familiar.

\subsubsection{Variable 2; Actividad Física y Salud}


Imagen 8. Interés por la actividad física y el deporte antes de la pandemia.

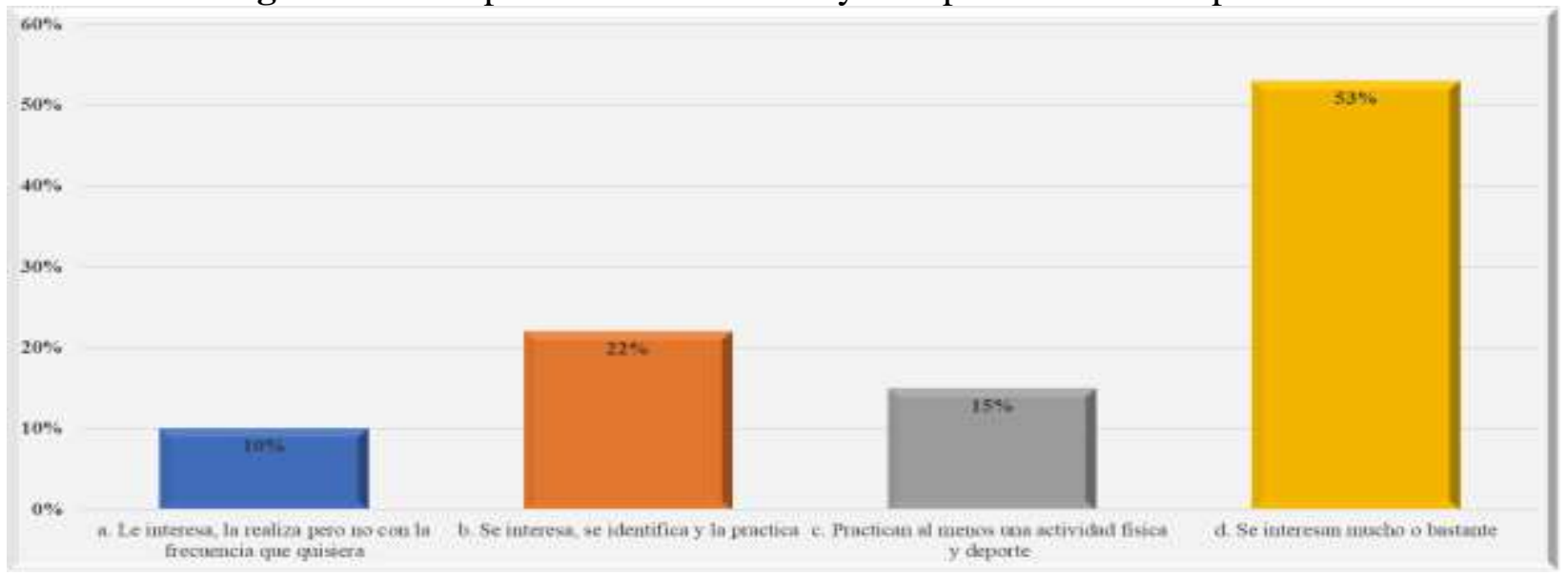

Fuente: Elaboración propia con base en los resultados obtenidos.

En la Imagen 8, se relaciona con el interés del estudiante por la actividad física y el deporte antes del confinamiento provocado por el COVID-19. El 53\% se interesan mucho o bastante, el $22 \%$ se interesa, se identifica y la lleva a cabo, el $15 \%$ practican al menos una actividad física y deporte y el $10 \%$ le interesa, la realiza, pero no con la frecuencia que quisiera.

Imagen 9. Actividad física y estilo de vida saludable durante el confinamiento

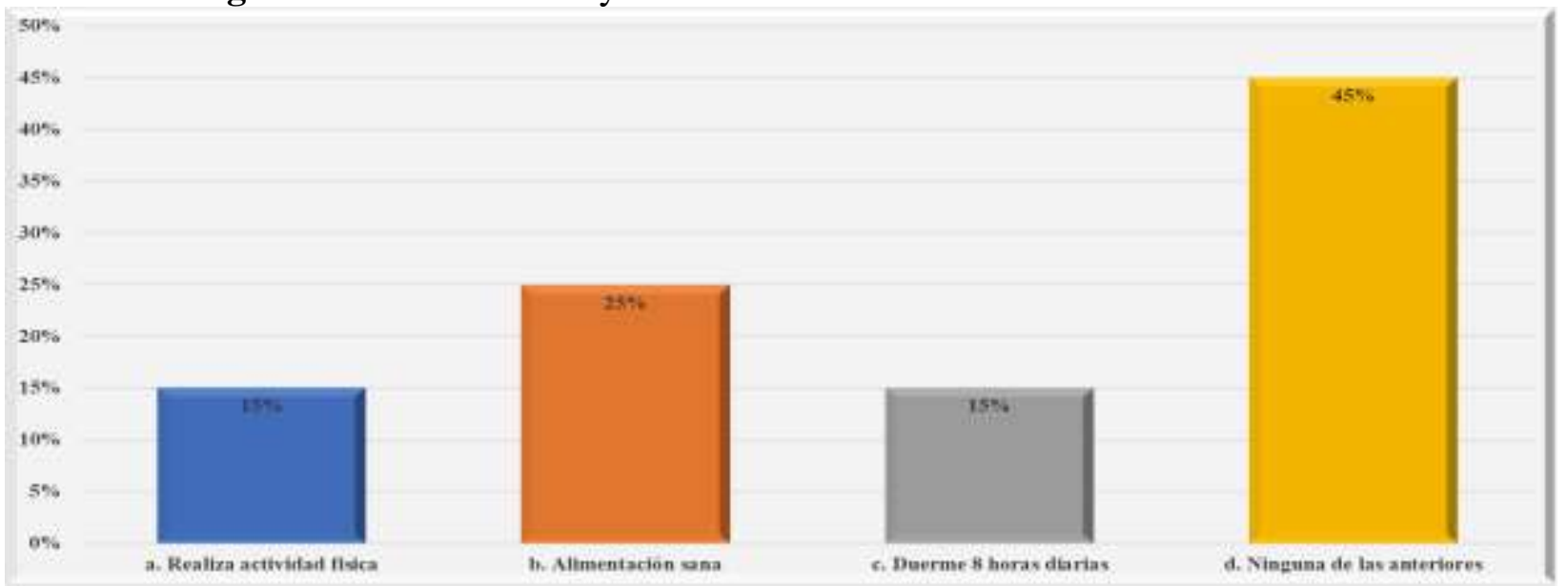

Fuente: Elaboración propia con base en los resultados obtenidos.

La Imagen 9, muestra información relacionada con la actividad física y el estilo de vida saludable del estudiante durante el confinamiento a partir del COVID-19. El 45\% de los estudiantes de la muestra analizada no realiza actividad fisica, no lleva una alimentación sana ni duerme 8 horas 
diarias, el $25 \%$ lleva una alimentación sana, hay una igualdad de resultados del $15 \%$ que mencionan que duerme 8 horas diarias y realiza actividad física.

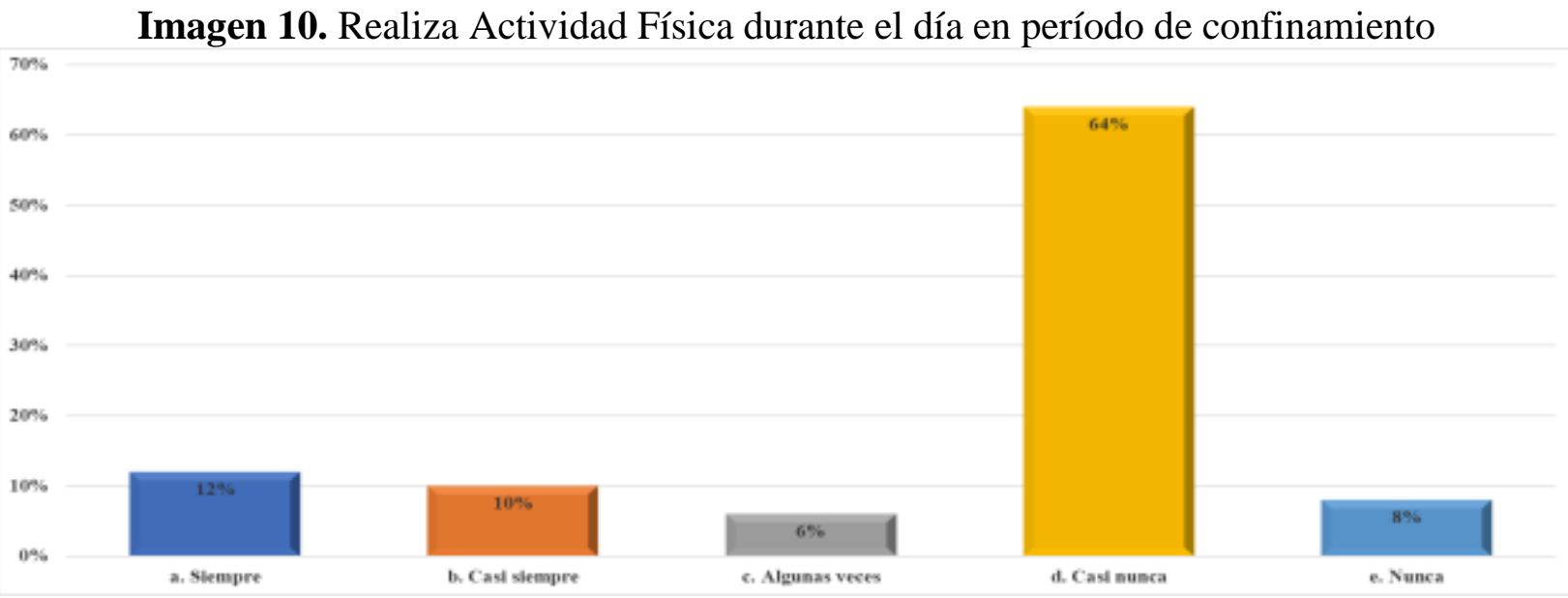

Fuente: Elaboración propia con base en los resultados obtenidos.

En la Imagen 10, muestra información relacionada con la realización de actividad física durante el período de confinamiento. El 64\% casi nunca la realiza, el 12\% menciona que siempre lo hace, el $10 \%$ casi siempre, el $8 \%$ nunca y el $6 \%$ algunas veces.

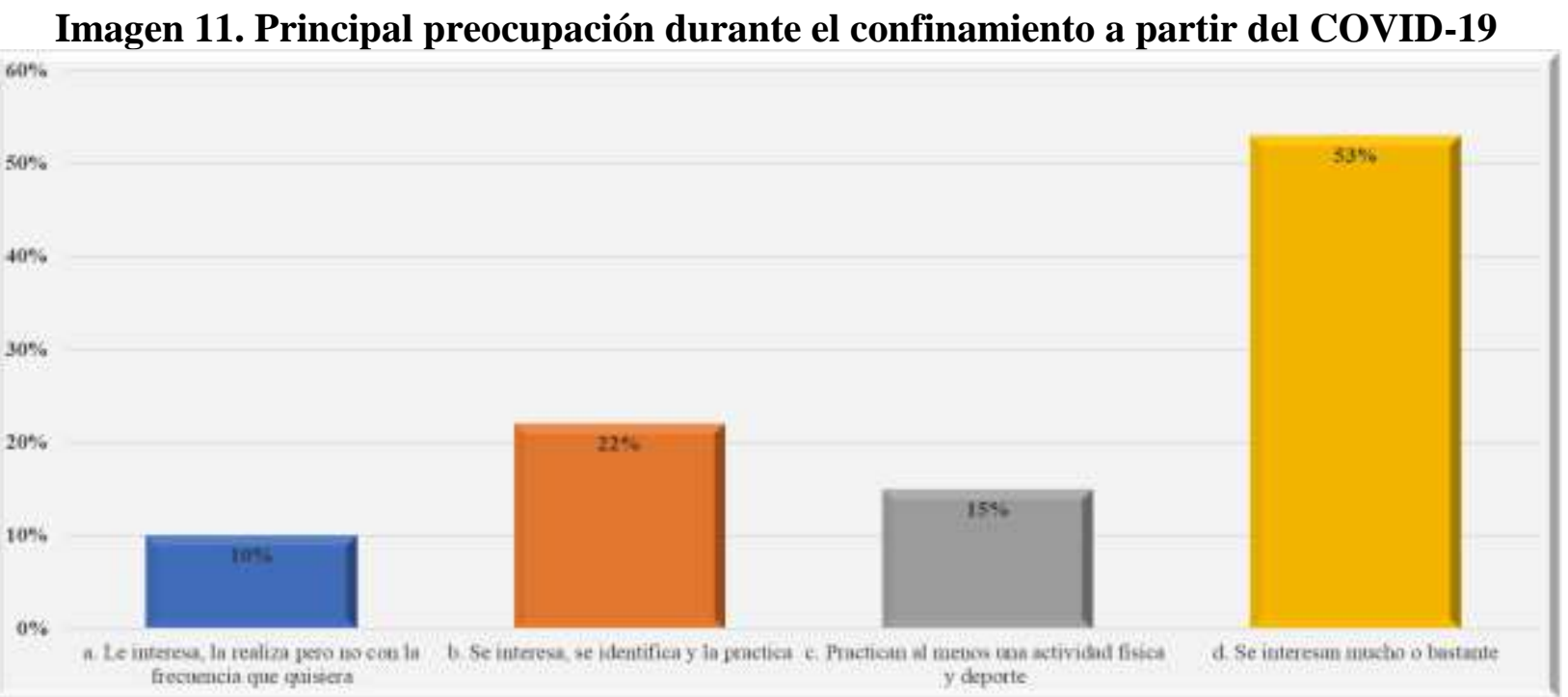

Fuente: Elaboración propia con base en los resultados obtenidos. 
En la Imagen 11, se relaciona con la principal preocupación durante el confinamiento a partir del COVID-19. Para el 43\% su principal preocupación es el cansancio, para el $20 \%$ su principal preocupación es no superar el aislamiento, para el $14 \%$ es la falta de convivencia familiar, para el $12 \%$ es la falta de convivencia con los amigos y para el $11 \%$ su salud.

\section{Discusión de resultados}

Dentro de los resultados obtenidos se muestra que dentro de la Variable 1; Estrés percibido en el estudiante universitario durante el confinamiento provocado por el COVID-19, más del 95\% de la muestra analizada tiene más de 12 meses de confinamiento y se observa que el $91 \%$ no había utilizado antes los espacios virtuales y ahora le dedican un tiempo de hasta 10 horas continuas para las actividades académicas. Los aspectos que influyen de forma negativa en su desempeño académico durante el confinamiento a partir del COVID-19 son: un ambiente desadaptado para realizar el trabajo desde casa, ya que no cuentan con espacios y las condiciones para realizarlo, sobrecarga de tareas, la ausencia del profesor para guiarlo, la convivencia con los amigos y la familia. Lo anterior, muestra que la principal preocupación del estudiante ha sido el tiempo que tienen en esas condiciones, cada vez más tareas, se siente cansado, el sedentarismo, la mala alimentación, falta de actividad física y la falta de ayuda motivacional o psicológica por parte de la universidad, hace ver que no cuentan con las habilidades para enfrentar la situación de tal forma que les provoca estrés y ansiedad y, esto repercute en su estado de salud físico y emocional.

Dentro de los resultados de la Variable 2; Actividad Física y Salud Laboral, los resultados muestran que el estudiante invierte muchas horas continuas a los espacios virtuales para atender sus actividades académicas y no tiene habitos de actividad física ni antes, ni durante el confinamiento, incrementando el sedentarismo, falta de un estilo de vida saludable y la ausencia de la convivencia con la familia o los amigos le ha provocado un cansancio, lo anterior hace suponer que posiblemente la falta de actividad fisica, el estilo de vida y los malos hábitos alimeticios le genera con mayor facilidad cansancio, estrés y problemas de salud. En las mismas opiniones mencionan que la empresa no se preocupa por el clima laboral en especial la salud de sus trabajadores, donde posiblemente se pudiera implementar un programa de actividad física y deporte con el fin de incidir en el mantenimiento y la mejora de la salud de sus trabajadores, dando como resultado la mejora de la calidad de vida. 


\section{Conclusiones}

El confinamiento supone que la persona realiza un menor esfuerzo físico en sus actividades diarias a favor del sedentarismo y, esto aunado a la falta de ejercicio físico en el entorno personal del estudiante ha favorecido el incremento de problemas de salud que lo llevan a tener una baja calidad de vida generando un bajo rendimiento y productividad. Los resultados aquí presentados, muestran la relación entre los niveles de estrés que manifiestan los estudiantes universitarios y la influencia de la actividad fisica como herramienta para la mejora de la salud psicológica del indivisuo que ayude en el rendimiento del estudiante en actividades académicas en espacios virtuales por causa de confinamiento (Xiang et al., 2020).

Si bien es cierto, la evidencia empírica hasta este momento aun es escasa, y no podemos afirmar que la Actividad Física podría disminuir los riesgos de infección por COVID-19, hay suficiente evidencia que demuestra que la Actividad Física planificada, estructurada, repetitiva y realizada por medio de pausaas activas tiene una relación con la mejora o el mantenimiento de uno o más de los componentes de la aptitud física, porque al realizarla de forma continua protege la salud del individuo, y se ha documentado la ayuda que brinda al control del estrés porque ayuda a mantener la función inmune, particularmente en personas sometidas a periodos largos de aislamiento y confinamiento como los presentados por la pandemia actual (Simpson, 2020) .

A modo de conclusión final y tomando en cuenta los planteamientos anteriores, se hace ver que la cuarentena obligatoria por el COVID-19, ha provocado cambios sustanciales en los niveles de actividad física entre los estudiantes durante el periodo de confinamiento, en la mayoría de los casos se prefirió un estado de sedentarismo y quienes sí practicaron lo realizaron en bien de cuidar su estado físico, por salud o entretenimiento y con la ayuda de recursos caseros. Al realizar un confinamiento por parte del estudiante, la falta de actividad física asociada a un asilamiento social le provoca estrés y ansiedad. Es importante que las universidades puedan incorporar un programa que contenga estrategias relacionadas con la Actividad Fisica, así como algunas recomendaciones de alimentación y medidas de pausas activas sumadas al descanso adecuado que ayuden a disminuir el estrés y la ansiedad del estudiante mediante actividades virtuales que ayuden en el mantenimiento de la condición física. Lo anterior, puede traer 
beneficios inmediatos en la reducción del riesgo de infección y mejorar la calidad de vida en familia, y otros en el mediano y largo plazo, como constituir un estilo de vida saludable como elemento protector de enfermedades crónicas e infecciosas.

Este estudio presenta varias limitaciones que sugieren vías de investigación futuras. La percepción del control del estrés a través de la actividad física para los estudiantes universitarios, es un resultado que debe analizarse para descartar la relatividad con base al género, edad y programa educativo. Los resultados aquí presentados comprueban el objetivo planteado en la investigación donde se destaca la importancia de que las universidades desarrollen e implementen un programa de ejercicio físico sistematizado y controlado en los programas de capacitación que ofrece la universidad, con el propósito de mejorar la salud y calidad de vida que ayude a desarrollar habilidades a sus estudiantes para un buen rendimiento en condiciones de confinamiento.

\section{Referencias}

Brooks, S. K., Webster, R. K., Smith, L. E., Woodland, L., Wessely, S., Greenberg, N., \& Rubin, G. J. (2020). The psychological impact of quarantine and how to reduce it: rapid review of the evidence. The Lancet, 395(10227), 912-920. https://doi. org/10.1016/S01406736(20)30460- 8

Giraldo Giraldo, V.A. (2021). Efectos del confinamiento en estudiantes universitarios: un análisis basado en diferencias de género. CIID Journal, 2(1), 509-521.

Ko, C. H., Yen, C. F., Yen, J. Y., \& Yang, M. J. (2006). Psychosocial impact among the public of the severe acute respiratory syndrome epidemic in Taiwan. Psychiatry and Clinical Neurosciences, 60(4), 397-403. https://doi.org/10.1111/j.1440- 1819.2006.01522.x

OMS, \& OPS. (2020). Smaps-Coronavirus-Es-Final17-Mar-20. 12 De Marzo 2020, 2019, 1-7. Organización Mundial de la Salud. (2020). Alocución de apertura del Director General de la OMS en la rueda de prensa sobre la COVID-19 celebrada el 11 de marzo de 2020. Discursos Del Director General de La OMS, 1. 
Ozamiz, N., Dosil, M., Picaza, M., \& Idoiaga, N. (2020). Niveles de estrés, ansiedad y depresión en la primera fase del brote del COVID-19 en una muestra recogida en el norte de España. Cad. Saúde Pública, 36(4), 1-10.

Ozamiz-etxebarria, N., Dosil-santamaria, M., Picaza-gorrochategui, M., \& Idoiagamondragon, N. (2020). Stress, anxiety, and depression levels in the initial stage of the COVID-19 outbreak in a population sample in the northern Spain Niveles de estrés, ansiedad y depresión en la primera fase del brote del COVID-19 en una muestra recogida en el norte de E. 36(4), 1-9. https://doi.org/10.1590/0102-311X00054020

Rodriguez-Morales, A. J., SánchezDuque, J. A., Hernández Botero, S., Pérez-Díaz, C. E., Villamil-Gómez, W. E., Méndez, C. A., LANCOVID-19. (2020). Preparación y control de la enfermedad por coronavirus 2019 (COVID-19) en América Latina. ACTA MEDICA PERUANA. https:// doi.org/10.35663/amp.2020.371.909

Ruiz-Frutos, C., y Gómez-Salgado, J. (2021). Efectos de la pandemia por COVID-19 en la salud mental de la población trabajadora. Archivos de Prevención de Riesgos Laborales, 24(1), 6-11

Simpson, R. J. (2020). American Collegue of sports medicine ACSM's. Ejercicio, inmunidad y la pandemia del COVID-19. Disponible en: https://www.acsm.org/blog-detail/acsmblog/2020/04/16/ejercicio-inmunidad-y-la-pandemia-del-covid-19

Villaquiran Hurtado, A. F., Ramos, O. A., Jácome, S. J., \& Meza Cabrera, M. del M. (2020). Actividad física y ejercicio en tiempos de COVID-19. CES Medicina, 34, 51-58.

World Health Organization. (2020). 2019 Novel Coronavirus (2019-nCoV): Strategic Preparedness and Response Plan. February, 28.

Xiang YT, Yang Y, Li W, Zhang L, Zhang Q, Cheung T, et al. (2020). Timely mental health care for the 2019 novel coronavirus outbreak is urgently needed. Lancet Psychiatry;7(3), 2289.

Yoon, M. K., Kim, S. Y., Ko, H. S., \& Lee, M. S. (2016). System effectiveness of detection, brief intervention and refer to treatment for the people with post-traumatic emotional distress by MERS: A case report of community-based proactive intervention in South Korea. International Journal of Mental Health Systems, 10(1), 1-5. https://doi.org/10.1186/s13033-016-0083-5 
Año 14 / Núm. 35 / Enero-junio 2021 Revista de Investigación Académica sin Frontera

https://revistainvestigacionacademicasinfrontera.unison.mx/index.php/RDIASF/article/view/394

Leon Bazan, M. J., \& Zolano Sánchez, M. L. (2021). Actividad física en época de confinamiento por COVID-19 para reducir los niveles de estrés en estudiantes universitarios. Revista De Investigación Académica Sin Frontera:

División De Ciencias Económicas Y Sociales, (35), 15. https://doi.org/10.46589/rdiasf.vi35.394

\section{CRIS - UNISON}

Sistema de Gestión de la Investigación

- Dialnet

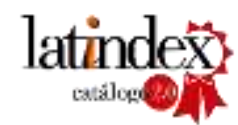

If uafacton

REDIB

Citefactor

$\underline{\text { Neliti - Indonesia's Research Repository }}$
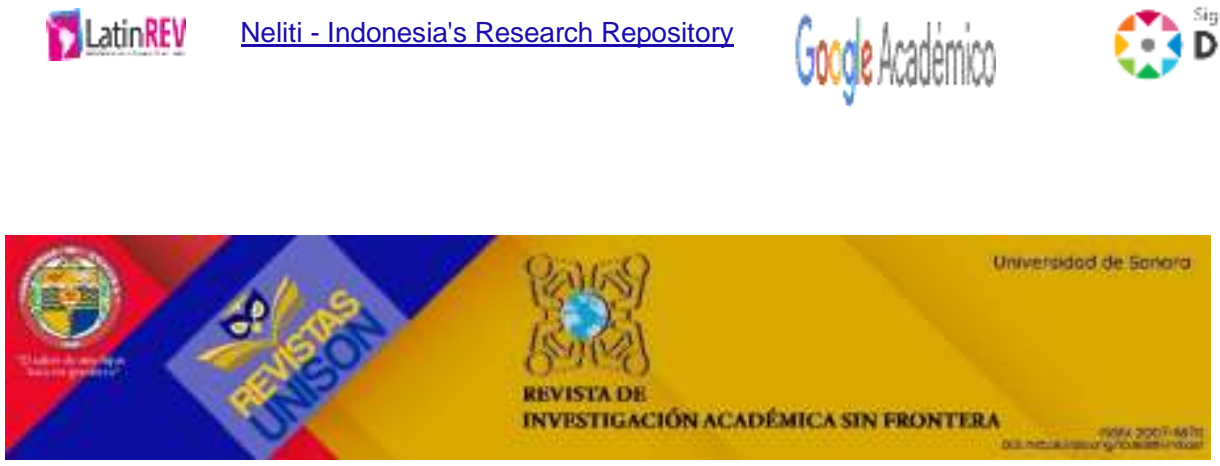